\title{
Special Issue on General Game Playing
}

\author{
Michael Thielscher
}

Published online: 16 December 2010

(C) Springer-Verlag 2010

Computer game playing has always been of special interest to Artificial Intelligence from the early days on. The cultural history of game-playing machines reaches even farther back than the advent of the modern computer. In the 18th century, an (allegedly) purely mechanical automaton, The Turk, outplayed almost any human chess player. The main reason for The Turk's popularity was that people considered human-level intelligence a basic prerequisite for being a good chess-player. This motivated the first AI researchers to regard chess-playing as an excellent benchmark for the creation of intelligent machines. But when in 1997 a computer finally defeated the then world chess champion in a six-game match, it was clear that progress in computer chess had little if any implications on how to build intelligent machines in general.

This insight has led to the recent interest in general game playing, which is concerned with systems that can learn to play just any game by being given nothing but the bare rules of that game. Figure 1 provides a schematic illustration of this principle. Because neither the specifics nor even the type of games to be played are known at programming time, a general game player cannot be endowed with fixed heuristics, tailor-made for one specific game. Rather, the player must be able to design a suitable strategy all by itself whenever it is asked to play a new game. Successful general game-playing programs can thus be seen as a new generation of AI systems that can be told what we want from them and that learn to carry out possibly radically different tasks without human intervention.

M. Thielscher $(\bowtie)$

School of Computer Science and Engineering, The University of New South Wales, Sydney NSW 2052, Australia

e-mail: mit@cse.unsw.edu.au
Research in general game playing actually began in the late 1960's with a computer program by Jacques Pitrat that was able to play any chess-like board game just by being given a procedural specification of the rules. Broad interest in this topic was sparked by the inauguration of the annual AAAI General Game Playing Competition in 2005, which came along with the attractive prize money of $\$ 10,000$. Since then, general game playing has rapidly developed into a well-established research topic. It is now regarded as one of the grand contemporary AI Challenges because it requires to combine methods from a number of diverse areas such as Knowledge Representation, Reasoning, Search, Planning, Computer Game Playing, and Learning. Players are being built around the world and participate regularly in international contests. Past winners of the AAAI competition came from the United States (2005, CLUNEPLAYER), Germany (2006, FLUXPLAYER), Iceland (2007 \& 2008, CADIAPLAYER), and France (2010, ARY). A series of biennial workshops on general game playing was established at IJCAI 2009, and AAAI 2010 featured the first ever technical session in the main program of a major AI conference with papers exclusively on this topic, contributing to a rapidly growing body of literature.

This first ever issue of a scientific journal on general game playing gives an excellent overview of the wide range of activities in this area by leading researchers around the world. In fact, each of the past AAAI champions is also an author for this special issue. I want to thank the editorial board of KI for the invitation to edit this special issue, the authors for their high-quality contributions, and the reviewers of all submitted papers for their efforts. Readers not yet familiar with general game playing are advised to have a look at Fig. 2 before they continue, since most articles make reference to the Game Description Language. 


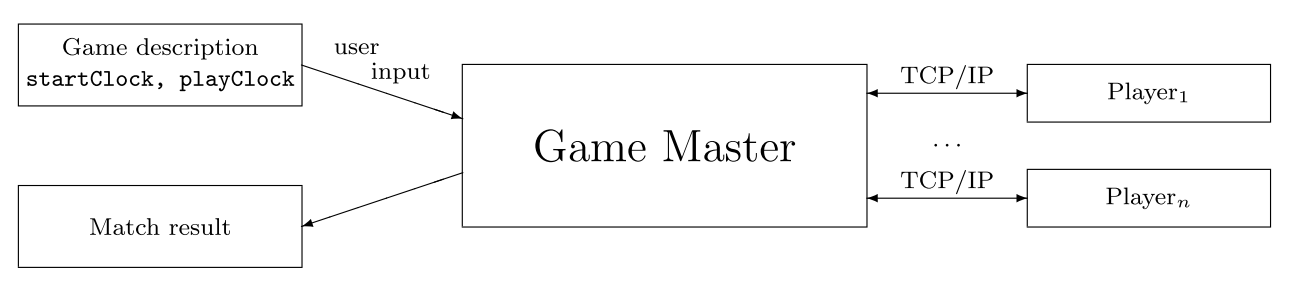

Fig. 1 To play an arbitrary game, its description is fed into a Game Master (GM) along with values for startClock (the time the players get to learn the new game) and playclock (the time for each move). The GM communicates rules and times to the $n \geq 1$ players and also assigns each player one of the $n$ individual roles in the game.
The match begins after startClock seconds, and then every playClock seconds the GM collects a move from each player and informs the players about each other's moves. Using the game rules the GM determines when a terminal position has been reached and the result of the match

The Game Description Language ( $G D L$ ) has been developed to describe the rules of arbitrary $n$-player games in a machine-processable language. It is similar to Prolog, including the negation-as-failure principle, but uses the standardised KIF notation, where variables are indicated by a leading "?" and clauses are written as $(<=$ head body $1 \ldots$ body $m$ ). Game descriptions use a few keywords with pre-defined meaning:

\begin{tabular}{|l|l|}
\hline $\begin{array}{l}\text { (role } r) \\
\text { init } \mathrm{f})\end{array}$ & $\begin{array}{l}\mathrm{r} \text { is a player } \\
\mathrm{f} \text { holds in the initial position }\end{array}$ \\
\hline $\begin{array}{l}\text { terminal } \\
(\text { goal } r \mathrm{n})\end{array}$ & $\begin{array}{l}\text { the current position is terminal } \\
\text { player } r \text { gets goal value } \mathrm{n}\end{array}$ \\
\hline
\end{tabular}

\begin{tabular}{|l|l|}
\hline $\begin{array}{l}\text { (true } \mathrm{f}) \\
\text { (next } \mathrm{f})\end{array}$ & $\begin{array}{l}\mathrm{f} \text { holds in the current position } \\
\mathrm{f} \text { holds in the next position }\end{array}$ \\
\hline $\begin{array}{l}\text { (legal } r \mathrm{~m}) \\
\text { (does } r \mathrm{~m})\end{array}$ & $\begin{array}{l}\text { player } r \text { has legal move } \mathrm{m} \\
\text { player } r \text { does move } \mathrm{m}\end{array}$ \\
\hline
\end{tabular}

A further standard predicate is (distinct $s t$ ), which means that its argument are syntactically distinct terms.

As an example, here is a complete axiomatisation of the game Tic-Tac-Toe.

1. First, we give arbitrary names to the two players.

$$
\text { (role xplayer) (role oplayer) }
$$

2. Initially, all cells of the $3 \times 3$-board are blank ("b"), and it's the first player's control.

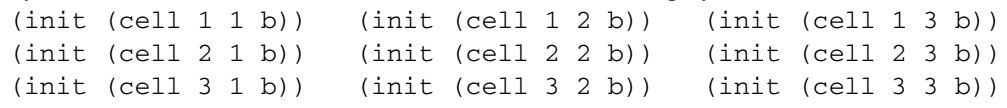

(init (control xplayer))

3. A player who has control can mark any cell that is currently blank; but you can do nothing if it's not your move.
$(<=$ (legal ?player (mark ?m ?n)) (true (control ?player)) (true (cell ?m ?n b)))
$(<=$ (legal xplayer noop)
$(<=$ (legal oplayer noop)
(true (control oplayer)))
(true (control xplayer)))

4. Changes are described in terms of how the next position depends on the moves and on what is currently true. Specifically, marking a cell changes the contents of that cell while all other cells remain unchanged, and the players take turn.
$(<=($ next $(\operatorname{cell} ? \mathrm{~m}$ ?n $\mathrm{x}))$
(does xplayer (mark ?m ?n)))
$(<=($ next $($ cell ?m ?n o))
(does oplayer (mark ?m ?n)))
$(<=($ next $($ cell ?m ?n ?c))
(true (cell ?m ?n ?c)) (does ?player (mark ?u ?v)) (distinct ?m ?u))
$(<=($ next $($ cell ?m ?n ?c))
(true (cell ?m ?n ?c)) (does ?player (mark ?u ?v)) (distinct ?n ?v))
$(<=$ (next (control xplayer)) (true (control oplayer)))
$(<=$ (next (control oplayer)) (true (control xplayer)))

5. A game ends when one player has completed a line, or when the board is full.
$1<=$ terminal
(line $\mathrm{x})$ )
$1<=$ terminal
(line o))
$(<=$ terminal (not open))
$(<=$ open (true (cell ?m ?n b)))
6. Finally, let the outcome of a game be given by the following goal values.

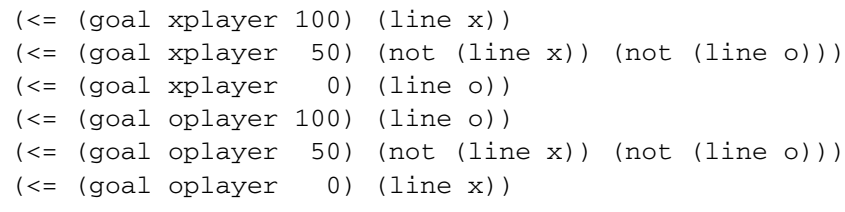

$(<=$ (line ?symbol) (true (cell ?m 1 ?symbol)) (true (cell ?m 2 ?symbol)) (true (cell ?m 3 ?symbol)))

$(<=$ (line ?symbol) (true (cell 1 ?n ?symbol)) (true (cell 2 ?n ?symbol)) (true (cell 3 ?n ?symbol))

$(<=($ line ?symbol) (true (cell $1 \quad 1$ ?symbol)) (true (cell $2 \quad 2$ ?symbol)) (true (cell $3 \quad 3$ ?symbol)))

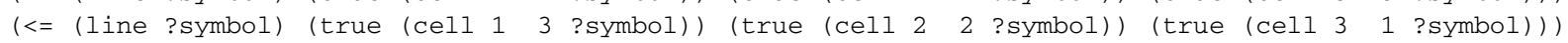

Fig. 2 A brief introduction to the Game Description Language 


\section{General Game Playing—Service by Stephan Schiffel}

This compilation of resources for general game playing should provide you with some starting points for reading, web search, and research. However, keep in mind that GGP is a highly diverse topic. Therefore, there are many publications in areas such as knowledge representation and reasoning, search, computer game playing, and learning that apply to GGP or are related to it.

\section{GGP in the Web}

The GGP web page of Stanford University

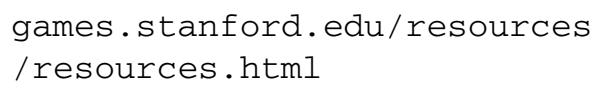

hosts the official specification of the Game Description Language (GDL), information about the international GGP competitions, and a mailing list. The mailing list is low traffic and features announcements about upcoming events such as competitions and workshops and publications about GGP.

Dresden University of Technology hosts

general-game-playing de

The web page contains a multitude of information about GGP, e.g., an extensive list of publications, source code of example players and libraries for writing an own general game player, information about GGP related events, and links to other GGP related web pages and projects.

\section{Conferences, Workshops, Competitions}

There is no special conference on General Game Playing. Papers about GGP are scattered over many different AI conferences. AAAI and ECAI added "general game playing" to their list of keywords recently and AAAI' 10 even featured the first session on General Game Playing.

The first workshop on General Intelligence in GamePlaying Agents (GIGA) was held at IJCAI'09 in Pasadena and featured 12 paper presentations. The next workshop will be held at the upcoming IJCAI'11 in Barcelona.

At the annual GGP world championship the top players of the world are pitted against each other. The competition serves as a platform for comparing state of the art players and for presenting GGP to a larger audience. There are other competitions on a smaller scale, e.g., in Stanford and Dresden and the German GGP Competition. They provide a first step for graduate students and other researchers that just started working on their player.

\section{Code \& Tools}

You can download starter code at both the Stanford and Dresden web pages mentioned above. Code is available in different programming languages (Java, C++, Lisp, Prolog) and ranges from simple players with a few hundred lines of code to frameworks containing search procedures, game analysis and other helpful components.

The GGP Server at

ggpserver.general-game-playing . de

can be used to test your player against a random player or other players that are online on a wide range of different games. Games that are played on the server are visualized for humans, such that you can see what is going on. The server also hosts competitions.

GameController is an offline version of the GGP server that can be easily used to test your player or run a series of experiments. It is open source and available for download at general-game-playing.de.

At

palamedes-ide.sourceforge.net

you can download an editor for game descriptions containing some game analysis and a step by step execution to simplify debugging of game descriptions. 